\title{
The Patient Experience: Symptoms and Impact of Dry Age-Related Macular Degeneration
}

Neil M. Schultz $\cdot$ Lydia Braunack-Mayer · Jason Schwartz ·

Luis Gaspar

Received: October 17, 2020 / Accepted: December 11, 2020 / Published online: January 29, 2021

(C) The Author(s) 2021

\section{ABSTRACT}

Introduction: No published literature systematically explores the dry age-related macular degeneration (AMD) patient experience. To inform the development of patient-reported outcome measures (PROMs), the important and relevant signs, symptoms, and impacts for patients with dry AMD were identified.

Methods: A holistic approach was used to capture, define, and organize the signs, symptoms, and impacts that are important to patients with dry AMD. Qualitative evidence was identified through a targeted literature review and clinician $(N=5)$ and patient $(N=20)$ interviews. The targeted review was expanded to include patients with AMD, as few studies specific to dry AMD were identified. The qualitative evidence was incorporated into a conceptual model that included the signs, symptoms, and impacts of dry AMD affecting the patient experience.

Supplementary Information The online version contains supplementary material available at https:// doi.org/10.1007/s40123-020-00325-y.

N. M. Schultz $(\varangle) \cdot J$. Schwartz

Astellas Pharma, Inc., Northbrook, IL, USA

e-mail: neil.schultz@astellas.com

L. Braunack-Mayer

IQVIA, Basel, Switzerland

L. Gaspar

IQVIA, Reading, UK
Results: Twenty-nine articles (dry AMD, $N=5$; general AMD, $N=24$ ) exploring health-related quality-of-life evidence in patients with AMD were identified. Concepts identified and included in the preliminary, literature-based model included signs and symptoms related to general vision loss and general impacts (e.g., dependency on others, poor spatial perception/mobility, difficulty reading, emotional affects). No concepts unique to dry AMD were identified. Interviewed clinicians refined the literaturebased model. Across all visual acuity severities, $\geq 80 \%$ of patients reported difficulty driving, reading, and completing activities of daily living, along with frustration and dependency on others; all patients reported blurred vision. The final model included 35 signs, symptoms, and impacts, with 19 considered salient.

Conclusions: To better understand the patient experience, we captured, defined, and organized signs, symptoms, and impacts into a dry AMD conceptual model. This model can aid in the development of PROMs reflecting the experience of patients with dry AMD.

Keywords: Conceptual model; Dry age-related macular degeneration; Dry AMD; Patientreported outcome measure; PROM 


\section{Key Summary Points}

No previously published literature systematically characterizes and reports the dry age-related macular degeneration (AMD) patient experience.

A holistic approach was used to capture, define, and organize the signs, symptoms, and impacts of dry AMD that are important to patients.

Thirty-five signs, symptoms, and impacts, 19 considered salient, were included in the final conceptual model, which can aid in the development of patient-reported outcome measures (PROMs) that accurately reflect what it's like to live with dry AMD from the patient perspective.

\section{DIGITAL FEATURES}

This article is published with digital features, including a summary slide, to facilitate understanding of the article. To view digital features for this article go to https://doi.org/10.6084/ m9.figshare.13365449.

\section{INTRODUCTION}

Age-related macular degeneration (AMD) is a leading cause of severe, irreversible vision impairment in developed countries, particularly in individuals aged 55 years and older $[1,2]$. The prevalence of AMD is estimated to increase from 196 million people in the year 2020 to 288 million by 2040 [3].

Age-related macular degeneration includes both wet, or neovascular, AMD and dry or atrophic AMD [4]. Although wet AMD accounts for a minority of AMD cases, most of the severe vision loss that occurs in patients with AMD occurs in those with wet AMD [5]. In contrast, dry AMD accounts for an estimated $80 \%$ of AMD cases and $10 \%$ of the severe vision impairment seen in patients with AMD [5]. With respect to available treatment options, antioxidant vitamins are the only American Academy of Ophthalmology-recommended option, the use of which may slow disease progression from earlier to later stages of dry AMD [5].

Regardless of the type of macular degeneration, AMD decreases quality of life (QoL) and leads to visual disability, adversely affecting activities of daily living such as the ability to drive, recognize faces, and use a computer [6]. Through visual impairment and visual loss, dry AMD impacts multiple aspects of the patient life experience.

As part of the 21st Century Cures Act, the US Food and Drug Administration (FDA) encouraged manufacturers to provide patient experience data, along with input from clinical experts, to help ensure that new treatments address patient needs as seen from the patient perspective [7]. Additionally, insights regarding the patient experience are increasingly being used by an array of stakeholders-along with other clinical data-to fully understand product value in healthcare, including applications in real-world clinical practice.

In reviewing the literature, patient-reported outcome measures (PROMs) used in published studies that enrolled patients with AMD are not necessarily specific to dry AMD and include both generic instruments (EuroQol-5D [EQ-5D] and 36-item Short Form Health Survey [SF-36]) and vision-specific instruments (National Eye Institute-Vision Function Questionnaire [NEIVFQ-25]) [8]. Although some vision-specific instruments are validated in patients with wet AMD [9], to our knowledge, none are validated in patients with dry AMD.

A critical understanding of the effect of dry AMD on QoL is needed, as well as the ability to assess this effect during routine ophthalmologic practice [9]. Therefore, this content analysis study aimed to capture, define, and organize information describing the patient dry AMD experience, including the distinct signs, symptoms, and impacts, to construct a holistic understanding of the patient experience. Results from this research led to the creation of 


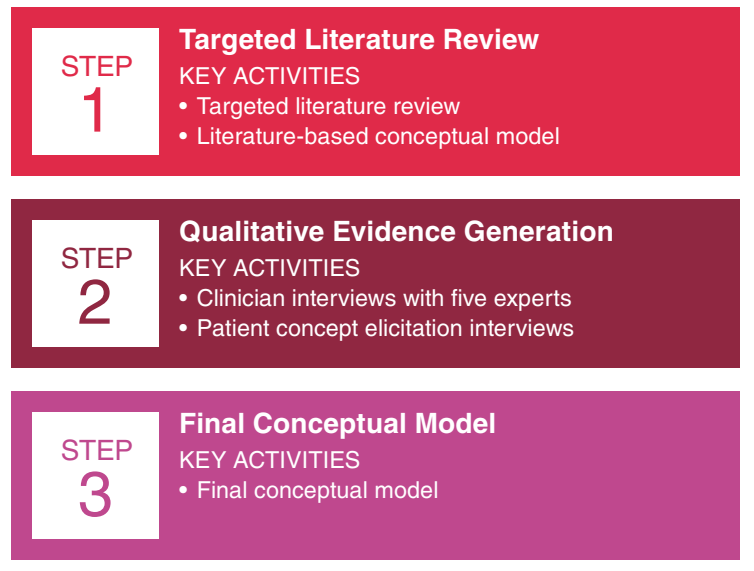

Fig. 1 Study design

the first conceptual model describing the patient dry AMD experience.

\section{METHODS}

A dry AMD conceptual model was developed using a three-step approach (Fig. 1). A preliminary conceptual model was developed based on results from a targeted literature review conducted to identify the most important signs, symptoms, and impacts related to the dry AMD patient experience. The literature-based model was refined and confirmed using input obtained from clinician and patient concept elicitation interviews, as recommended in the 2009 FDA Guidance for Industry on the use of PROMs [10].

\section{Literature Review}

The PubMed, Cochrane, PsycINFO, and Embase databases were searched to identify evidence on health-related QoL domains, including signs and symptoms, functional status, general health perception, and overall QoL. An initial search targeting dry AMD identified 11 articles. To ensure comprehensive identification of relevant literature, the targeted-literature review was expanded to include the broader AMD population. Searches were limited to publications in English that were available after 1995 (Table 1; Appendix).
Table 1 Literature review scope

\begin{tabular}{|c|c|c|}
\hline Scope & Inclusion criteria & Exclusion criteria \\
\hline $\begin{array}{l}\text { Population of } \\
\text { interest }\end{array}$ & Patients with AMD & $\begin{array}{l}\text { Patients with other } \\
\text { diseases or eye } \\
\text { conditions, or } \\
\text { non-relevant } \\
\text { comorbidities }\end{array}$ \\
\hline $\begin{array}{c}\text { Interventions } \\
\text { of interest }\end{array}$ & $\begin{array}{l}\text { All studies, } \\
\text { irrespective of } \\
\text { patients receiving } \\
\text { therapy or not }\end{array}$ & Not applicable \\
\hline $\begin{array}{l}\text { Outcomes of } \\
\text { interest }\end{array}$ & $\begin{array}{l}\text { Concepts (i.e., signs, } \\
\text { symptoms, and } \\
\text { impacts of a } \\
\text { disease and its } \\
\text { treatments) }\end{array}$ & $\begin{array}{l}\text { Literature not } \\
\text { including an } \\
\text { outcome of } \\
\text { interest }\end{array}$ \\
\hline $\begin{array}{c}\text { Sources of } \\
\text { interest }\end{array}$ & $\begin{array}{l}\text { Patient interview } \\
\text { studies } \\
\text { Expert guidelines } \\
\text { Instrument } \\
\text { development } \\
\text { Epidemiology } \\
\text { studies } \\
\text { Systematic reviews }\end{array}$ & Other sources \\
\hline
\end{tabular}

$A M D$ age-related macular degeneration

\section{Qualitative Evidence Generation}

\section{Clinician Interviews}

Clinicians were identified and recruited from different regions in the United States who met the following criteria: associated with leading eye-specialty treatment centers and/or research institutions, expertise in treating patients with dry AMD (50-200 patients per month), and a record of published research in AMD. Clinicians reviewed the literature-based model and provided input on the types and severity of dry AMD signs/symptoms, as well as the consequences and degree of disturbance of dry AMD on patient lives. 


\section{Patient Interviews}

Concept elicitation interviews were conducted with a representative sample of patients recruited via different methodologies, including physician recruitment, patient advocacy groups, social media platforms, and recruitment partners. All patients provided informed consent and were residents of the continental United States, aged 50 years or older, had a diagnosis of dry AMD in at least one eye, and had visual acuity of 20/40 or lower in both eyes. Patients interviewed had mild (i.e., visual acuity between $20 / 40$ and 20/80 in both eyes), moderate (visual acuity between 20/80 and 20/200 in at least one eye), or severe (visual acuity equal to or below 20/200 in at least one eye) impairment. Patients were excluded if they were diagnosed with wet AMD in either eye or had any ophthalmologic condition, medication use, or treatment that rendered them unsuitable for inclusion. Ophthalmologic conditions that could render the patient unsuitable for inclusion encompassed the following: retinal disease affecting the macula (e.g., inherited retinal diseases, diabetic macular edema, pathologic myopia, retinal detachment), ocular inflammation (e.g., uveitis), ocular malignancy, or any ocular conditions affecting central vision and/ or retinal imaging. Medications and treatments that excluded patients included anti-vascular endothelial growth factor therapy, intraocular or refractive surgery, an ocular implant, gene transfer or cell transplantation therapy, or any ocular drug in the context of a previous clinical trial in the past 12 weeks. Patients with a mental disability or significant mental illness, legal incapacity, limited legal capacity, or any other lack of fitness that, in the opinion of the screener, would preclude the participant's participation in or ability to complete the study were also excluded.

All patients were screened over the phone, consented verbally, and provided a copy of their consent via email or mail. Following consent, patients were contacted to schedule a phone interview which was estimated to last $60 \mathrm{~min}$. The interviews were conducted to provide a direct perspective on living with dry AMD and to allow for a better understanding of patients' terminology for describing dry AMD and treatment concepts. An interview guide was used by the interviewers to ensure consistency in interview content. The interviews were conducted by two trained interviewers (LG [PhD], LB-M [MSc]) with experience in conducting individual patient interviews. Interviewers shared with participating patients the reasons for performing the interview and their interest in the research topic. With the patient's consent, other interviewers were permitted to listen to the call for training or quality assurance purposes. Patient responses to discussion questions were captured by a moderator on a de-identified copy of the interview guide as well as de-identified worksheets for reported signs, symptoms, and impacts. All interviews were recorded with patient consent and were transcribed; transcripts were not shared with patients.

Four waves of interviews, each consisting of five patients, were conducted by the two trained interviewers [7]. During the session, patients were asked a set of open-ended questions with probes to explore their experience with dry AMD. Patients were asked about their first experience with the condition, how their experience may have changed over time, and current signs, symptoms, and impacts of the condition and its treatments. Additionally, patients were asked to rate the degree of disturbance the symptoms and impacts had on their daily lives.

For each concept identified, interviewers asked patients how disturbing the symptom or impact was or is to their life using a 0-10 scale, with 0 being "does not disturb" and 10 being "greatly disturbs." The interview approach followed the recommendations provided by the International Society for Pharmacoeconomics and Outcomes Research Good Research Practices Task Force $[11,12]$. No repeat interviews were conducted, and patients did not provide feedback on study findings.

\section{Treatment of Study Data}

De-identified transcripts of patient interviews were coded using ATLAS.ti. Two coders coded the transcripts, identifying patient descriptions and disturbance ratings of signs, symptoms, and impacts. Patients could report disturbance 
ratings for multiple time periods throughout their disease progression. In these cases, the highest number was reported during the coding process to capture the worst extent of symptom or impact disturbance.

\section{Concept Saturation}

Saturation of concepts (i.e., the point at which additional patient interviews did not contribute unique concepts or new information) was assessed to ensure adequate sample size, given that there are no suitable methods for power calculations in qualitative interview studies. To evaluate saturation, the codes derived from the second wave of interviews were compared with the codes from the first wave of interviews to determine whether any new information at the concept level was present in the second group. If new concepts appeared in the second group, saturation was not achieved. The comparison was repeated for the next wave of interviews, and the point at which saturation was achieved was identified.

\section{Salience Analysis}

The study considered both the number of patients mentioning a concept and the average disturbance rating to identify the most salient signs, symptoms, and impacts of dry AMD. A concept was deemed salient if at least $50 \%$ of patients mentioned the concept and the average disturbance rating was at least 5.0.

\section{Human Subject Approval}

The study was conducted in accordance with ethical principles that have their origin in the Declaration of Helsinki and are consistent with Good Clinical Practice and applicable regulatory requirements. The study was conducted in accordance with the regulations of the United States FDA as described in 21 CFR 50 and 56, applicable laws, and institutional review board requirements. Ethics approval was obtained from the New England Institutional Review Board (NEIRB), 197 First Avenue, Suite 250, Needham, MA 02494.

\section{Final Conceptual Model}

Following interviews, the signs, symptoms, and impacts included in the literature-based conceptual model were refined based on physician and patient responses and were incorporated into a final conceptual model.

\section{RESULTS}

\section{Targeted Literature Review and Preliminary Conceptual Model}

The targeted literature review, conducted in December 2018, identified 29 articles exploring health-related QoL evidence in patients with AMD that provided inputs for the literaturebased conceptual model (Fig. 2). Five articles specifically focused on dry AMD only; no concepts exclusive to dry AMD were identified.

Patient experience data identified from the literature review and included in the initial conceptual model were confirmed with an analysis of data obtained from patient blogs.

\section{Clinician Interviews and Model Refinement}

Five clinicians reviewed the initial literaturebased model and recommended refinements to the conceptual model (Fig. 3). The clinicians refined the existing concepts and suggested the addition of new concepts and exclusion of others to accurately capture the patient experience. Key impacts highlighted by all clinicians included difficulty reading and driving, stress, anxiety, and frustration.

\section{Patient Concept Elicitation Interviews and Model Refinement}

Twenty patients with dry AMD participated in the concept elicitation interviews; no patients refused to participate in the interviews following confirmation of eligibility and diagnosis. Interviewed patients skewed toward elderly (mean age 69 years, range 51-83 years), white, and female patients; most had moderate eye 


\begin{tabular}{|c|c|}
\hline $\begin{array}{l}\text { SIGNS \& SYMPTOMS } \\
\text { - Blurred vision } \\
\text { - Progressive vision loss } \\
\text { - Restricted visual fields } \\
\text { - Night blindness } \\
\text { - Defective color vision } \\
\text { - Poor light adaptation } \\
\text { - Ocular pain } \\
\text { - Poor depth perception } \\
\text { - Visual hallucinations } \\
\text { - Light flashes }\end{array}$ & $\begin{array}{ll}\begin{array}{l}\text { IMMEDIATE IMPACTS } \\
\text { - Dependency on other }\end{array} & \begin{array}{c}\text { - Inability to play sports } \\
\text { people }\end{array} \\
\text { - Poor spatial perception } & \text { activities } \\
\text { and mobility } & \text { - Embarrassment } \\
\text { - Difficulty reading } & \text { - Lack of confidence } \\
\text { - Difficulty completing } & \text { - Dissatisfaction with } \\
\text { activities of daily living } & \text { HCP } \\
\text { - Difficulty driving } & \text { - Need to wear glasses } \\
\text { - Worry about disease } & \text { - Shame about disease } \\
\text { and future } & \text { condition } \\
\text { - Frustration } & \text { - Lack of motivation } \\
\text { - Stress and anxiety } & \text { - Falls/accidents }\end{array}$ \\
\hline
\end{tabular}

Fig. 2 Initial dry AMD conceptual model based on targeted literature review. Note: Concepts are based on AMD literature search and not solely on dry AMD. No concepts exclusive to dry AMD were found in the literature. Concepts are listed by order of number of

disease (Table 2). There was a trend for decreasing visual acuity in the best-seeing eye, with decreasing visual acuity in the worst-seeing eye.

Six new concepts were identified that were not included in the literature-based model. No new concepts emerged in waves 3 or 4 , demonstrating that symptom and concept saturation were achieved in wave 2 .

The patient concept elicitation interviews identified blurred vision as a key symptom that was reported by all patients regardless of disease severity (Table 3). Patient quotes describing the symptoms of dry AMD are provided in articles mentioning the concept. $A M D$ age-related macular degeneration, $H C P$ healthcare provider

Appendix 1 of the Electronic Supplementary Material (ESM) (Supplementary Table 1).

Results from the disturbance analysis identified progressive vision loss, blurred vision, difficulty seeing in low light, and poor light/dark adaptation as the most bothersome symptoms reported by $80 \%$ or more of patients. The most salient signs and symptoms were blurred vision, difficulty seeing in low light, progressive vision loss, poor depth perception, poor light/dark adaptation, distorted vision, difficulty seeing contrasts or the reporting of items as appearing "washed out," and loss of central visual field/central blind spot. 


\begin{tabular}{|c|c|}
\hline $\begin{array}{l}\text { SIGNS \& SYMPTOMS } \\
\text { - Blurred vision } \\
\text { - Progressive vision loss } \\
\text { - Loss of central visual } \\
\text { field/central blind spot } \\
\text { - Difficulty seeing in } \\
\text { low-light environment } \\
\text { - Restricted visual fields } \\
\text { - Night blindness } \\
\text { - Poor light/dark } \\
\text { adaptation } \\
\text { - Poor contrast } \\
\text { vision/things appear } \\
\text { washed out } \\
\text { - Ocular pain } \\
\text { - Poor depth perception } \\
\text { - Defective color vision } \\
\text { - Visual hallucinations } \\
\text { (typically occurs when } \\
\text { there is significant central } \\
\text { vision loss with both eyes) } \\
\text { - Light flashes/floaters } \\
\text { - Distorted vision - straight } \\
\text { lines appear wavy }\end{array}$ & $\begin{array}{ll}\text { IMMEDIATE IMPACTS } & \\
\text { - Difficulty driving } & \text { - Inability to play sperts } \\
\text { - Dependency on other } & \text { ef engage in physical } \\
\text { people } & \text { activities } \\
\text { - Poor spatial perception } & \text { - Embarrassment } \\
\text { and mobility } & \text { - Lack of confidence } \\
\text { - Difficulty reading } & \text { - Dissatisfaction with } \\
\text { - Difficulty completing } & \text { HCP } \\
\text { activities of daily living } & \text { - Need to wear glasses } \\
\text { - Worry about disease } & \text { - Shame about disease } \\
\text { and future/fear of } & \text { condition } \\
\text { disease progressing } & \text { - Lack of motivation } \\
\text { - Frustration } & \text { - Falls/accidents } \\
\text { - Stress and anxiety } & \end{array}$ \\
\hline
\end{tabular}

Fig. 3 Revised dry AMD conceptual model based on input from clinician interviews. Red text: New concepts mentioned by the clinicians. Bold text: Concepts most important to patients as mentioned by the clinicians. Strikethrough: Concept mentioned by at least two

No notable severity-specific symptoms were identified, and no frequency of mention trend was observed across disease severities.

Impacts mentioned by more than $80 \%$ of patients included difficulty driving, reading, and completing activities of daily living, as well as frustration and dependency on other people. These impacts were largely attributed to the most salient signs and symptoms mentioned by patients. For example, because of blurred vision and difficulty seeing in low-light environments, patients described having difficulty driving and reading. Patient quotes describing the impacts clinicians as not characteristic of the patient experience of dry AMD. AMD age-related macular degeneration, $H C P$ healthcare provider

of dry AMD are provided in Appendix 1 of the ESM (Supplementary Table 2).

The most disturbing impacts to patients were eye strain, difficulty reading, and financial difficulties. The most salient impacts were difficulty reading and driving, frustration, dependency on other people, difficulty completing activities of daily living, worry about their disease and the future, inability to or limited participation in social and leisure activities, stress and anxiety, lack of confidence, poor spatial perception and mobility, and depression. 
Table 2 Patient characteristics: concept elicitation interviews

\begin{tabular}{ll}
\hline Characteristic & All patients $(\boldsymbol{N}=\mathbf{2 0})$ \\
\hline Age, years, mean (min-max) & $69(51-83)$ \\
Female, $n$ (\%) & $12(60)$ \\
Race, $n$ (\%) & $9(45)$ \\
White & $5(25)$ \\
Black/African American & $3(15)$ \\
Hispanic & $1(5)$ \\
Pacific Islander & $2(10)$ \\
Not available & $8(40)$ \\
Number of eyes affected, $n(\%)$ & $12(60)$ \\
One & \\
Both & $5(25)$ \\
Disease severity, $n(\%)^{\mathrm{a}}$ & $10(50)$ \\
Mild & $5(25)$ \\
Moderate & \\
Severe & \\
Employment status, $n(\%)$ & \\
Employed & \\
Not available & \\
\hline
\end{tabular}

${ }^{a}$ Disease severity defined as follows: mild, visual acuity of $20 / 40$ to $20 / 80$ in both eyes; moderate, visual acuity of $20 / 80$ to $20 / 200$ in worst-seeing eye; severe: visual acuity of $20 / 200$ or less in worst-seeing eye

\section{Final Conceptual Model}

The final conceptual model included 35 signs, symptoms, and impacts, 19 of which were mentioned by at least $50 \%$ of patients and received a disturbance rating of 5 or higher on average, and were therefore considered salient (Fig. 4). Concepts not identified in the literature-based model that were noted by patients and included in the final model were ocular dryness, itching, and irritation; headache; disorientation; difficulty recognizing faces; eye strain; and slow vision adjustment in the morning. Concepts included in the literaturebased model, but not mentioned by patients and therefore excluded from the final model, included night blindness, ocular pain, gastrointestinal distress, dissatisfaction with healthcare provider, shame about disease condition, and lack of motivation.

\section{DISCUSSION}

To better understand the dry AMD patient experience, we captured, defined, and organized information on the distinct signs, symptoms, and impacts of disease using a rigorous threestep analysis. Results from this exercise led to the development of the first known conceptual model in dry AMD. Our approach provided a comprehensive review of the patient experience and identified connections between salient impacts and specific signs and symptoms noted by patients. Of the 35 signs, symptoms, and impacts included in the final conceptual model, 19 were mentioned by the majority of patients and received a disturbance rating of 5 or higher. As such, this conceptual model can be used to support the development of a fit-for-purpose clinical outcomes assessment.

Research that identifies the key effects of dry AMD on the patient experience, including the signs, symptoms, and impacts of disease, can enhance regulatory decision-making and serve as a resource for regulators, payers, clinicians, and patients [7]. Examples of label claims that incorporate patient-reported outcomes in ophthalmology include alcaftadine for ocular itching [13] and ciclosporin for ocular symptoms [14] in the United States, and ranibizumab for vision-related functioning [15] in Europe. However, few data are available in the published literature on conceptual model development or steps used to develop the patient-reported outcome strategy that supports these claims.

Results from a review of the literature identified several validated PROMs used in assessing QoL in patients with wet AMD [9], but no measures that specifically addressed QoL or the effect of symptoms on QoL in patients with $d r y$ AMD. To the extent that the two diseases differ 
Table 3 Signs and symptoms and immediate and general impacts of dry AMD mentioned in patient interviews: overall and by disease severity

\begin{tabular}{|c|c|c|c|c|}
\hline & \multicolumn{4}{|c|}{ Patients, N (\%) } \\
\hline & \multirow[b]{2}{*}{$\begin{array}{l}\text { Overall } \\
(\mathrm{N}=20)\end{array}$} & \multicolumn{3}{|c|}{ Disease severity } \\
\hline & & $\begin{array}{l}\text { Mild } \\
(n=5)\end{array}$ & $\begin{array}{c}\text { Moderate } \\
(n=10)\end{array}$ & $\begin{array}{c}\text { Severe } \\
(n=5)\end{array}$ \\
\hline \multicolumn{5}{|l|}{ Signs and symptoms } \\
\hline Blurred vision & $20(100)$ & $5(100)$ & $10(100)$ & $5(100)$ \\
\hline Difficulty seeing in low-light environment & $17(85)$ & $4(80)$ & $9(90)$ & $4(80)$ \\
\hline Progressive vision loss & $17(85)$ & $4(80)$ & $8(80)$ & $5(100)$ \\
\hline Poor depth perception & $16(80)$ & $4(80)$ & $9(90)$ & $3(60)$ \\
\hline Distorted vision - straight lines appear wavy & $15(75)$ & $3(60)$ & $8(80)$ & $4(80)$ \\
\hline Poor contrast vision/things appear washed out & $15(75)$ & $3(60)$ & $8(80)$ & $4(80)$ \\
\hline Poor light/dark adaptation & $15(75)$ & $4(80)$ & $8(80)$ & $3(60)$ \\
\hline Light flashes/floaters & $12(60)$ & $3(60)$ & $7(70)$ & $2(40)$ \\
\hline Loss of central visual field/central blind spot & $11(55)$ & $3(60)$ & $6(60)$ & $2(40)$ \\
\hline Defective color vision & $9(45)$ & $1(20)$ & $5(50)$ & $3(60)$ \\
\hline Headache & $5(25)$ & $2(40)$ & $0(0)$ & $3(60)$ \\
\hline Ocular dryness, itching, or irritation & $5(25)$ & $2(40)$ & $2(20)$ & $1(20)$ \\
\hline Visual hallucinations & $5(25)$ & $1(20)$ & $4(40)$ & $0(0)$ \\
\hline Restricted visual fields & $4(20)$ & $0(0)$ & $2(20)$ & $2(40)$ \\
\hline
\end{tabular}

in terms of presentation and population, and that clinical outcome instruments designed for the two separate populations are deemed valuable, our research could therefore prove seminal when used to elucidate the impact of dry AMD and its symptoms on QoL.

Change in visual acuity is often used to assess treatment response in patients with AMD, in both clinical trials and clinical practice [9]. However, visual acuity is a clinical measurement and physician-reported; therefore, it does not assess change from the patient perspective [9] or provide insight into the psychological effects on patients. Results from a recently completely literature review of PROMs in ophthalmology concluded that the patient preferences identified using these instruments in routine practice may surprise healthcare providers and policymakers, ultimately transforming the way patients seen in ophthalmology practices are treated [16].

The utilization of non-disease-specific QoL instruments may be meaningful for economic evaluation, but they often lack the sensitivity for measuring the effect of signs, symptoms, and impacts on patients with eye diseases [16]. Additionally, non-vision-specific assessments of QoL do not allow patients to document the 
Table 3 continued

\begin{tabular}{|c|c|c|c|c|}
\hline & \multicolumn{4}{|c|}{ Patients, N (\%) } \\
\hline & \multirow[b]{2}{*}{$\begin{array}{l}\text { Overall } \\
(\mathrm{N}=20)\end{array}$} & \multicolumn{3}{|c|}{ Disease severity } \\
\hline & & $\begin{array}{l}\text { Mild } \\
(n=5)\end{array}$ & $\begin{array}{l}\text { Moderate } \\
(n=10)\end{array}$ & $\begin{array}{c}\text { Severe } \\
(n=5)\end{array}$ \\
\hline \multicolumn{5}{|l|}{ Immediate impacts } \\
\hline Difficulty driving & $19(95)$ & $5(100)$ & $10(100)$ & $4(80)$ \\
\hline Difficulty reading & $19(95)$ & $5(100)$ & $9(90)$ & $5(100)$ \\
\hline Difficulty completing activities of daily living & $18(90)$ & $5(100)$ & $9(90)$ & $4(80)$ \\
\hline Frustration & $18(90)$ & $3(60)$ & $10(100)$ & $5(100)$ \\
\hline Dependency on other people & $17(85)$ & $3(60)$ & $9(90)$ & $5(100)$ \\
\hline $\begin{array}{l}\text { Worry about disease and future/fear of disease } \\
\text { progressing }\end{array}$ & $15(75)$ & $3(60)$ & $8(80)$ & $4(80)$ \\
\hline Stress and anxiety & $13(65)$ & $2(40)$ & $8(80)$ & $3(60)$ \\
\hline Lack of confidence & $11(55)$ & $2(40)$ & $6(60)$ & $3(60)$ \\
\hline Poor spatial perception and mobility & $11(55)$ & $1(20)$ & $7(70)$ & $3(60)$ \\
\hline Need to wear glasses & $9(45)$ & $2(40)$ & $4(40)$ & $3(60)$ \\
\hline Inability to play sports or engage in physical activities & $6(30)$ & $1(20)$ & $3(30)$ & $2(40)$ \\
\hline Eye strain & $7(35)$ & $1(20)$ & $2(20)$ & $4(80)$ \\
\hline Falls/accidents & $6(30)$ & $2(40)$ & $3(30)$ & $1(20)$ \\
\hline Difficulty recognizing faces & $5(25)$ & $4(80)$ & $0(0)$ & $1(20)$ \\
\hline Disorientation & $4(20)$ & $1(20)$ & $2(20)$ & $1(20)$ \\
\hline Embarrassment & $4(20)$ & $1(20)$ & $3(30)$ & $0(0)$ \\
\hline Vision slow to adjust in morning & $2(10)$ & $0(0)$ & $2(20)$ & $0(0)$ \\
\hline \multicolumn{5}{|l|}{ General impacts } \\
\hline $\begin{array}{l}\text { Inability or limitation in participation of social and } \\
\text { leisure activities }\end{array}$ & $15(75)$ & $3(60)$ & $8(80)$ & $4(80)$ \\
\hline Depression & $10(50)$ & $2(40)$ & $5(50)$ & $3(60)$ \\
\hline $\begin{array}{l}\text { Less productivity at work/unemployment/having to } \\
\text { switch jobs }\end{array}$ & $3(15)$ & $3(60)$ & $0(0)$ & $0(0)$ \\
\hline Financial difficulties & $2(10)$ & $1(20)$ & $1(10)$ & $0(0)$ \\
\hline
\end{tabular}

Concepts are ordered by number of mentions in patient interviews. Bold text: Symptoms/impacts mentioned by $\geq 50 \%$ of patients

$A M D$ age-related macular degeneration 


\begin{tabular}{|c|c|}
\hline $\begin{array}{l}\text { SIGNS \& SYMPTOMS } \\
\text { - Progressive vision loss } \\
\text { - Blurred vision } \\
\text { - Difficulty seeing in } \\
\text { low-light environment } \\
\text { - Poor light/dark } \\
\text { adaptation } \\
\text { - Distorted vision - } \\
\text { straight lines appear } \\
\text { wavy } \\
\text { - Poor depth perception } \\
\text { - Defective color vision } \\
\text { - Poor contrast } \\
\text { vision/things appear } \\
\text { washed out } \\
\text { - Loss of central visual } \\
\text { field/central blind spot } \\
\text { - Light flashes/floaters } \\
\text { - Restricted visual fields } \\
\text { - Ocular dryness, itching } \\
\text { and irritation } \\
\text { - Headache } \\
\text { - Visual hallucinations } \\
\text { (typically occurs when } \\
\text { there is significant central } \\
\text { vision loss with both eyes) }\end{array}$ & $\begin{array}{ll}\text { IMMEDIATE IMPACTS } & \\
\text { - Difficulty reading } & \text { - Stress and anxiety } \\
\text { - Frustration } & \text { • Eye strain } \\
\text { - Difficulty driving } & \text { - Need to wear glasses } \\
\text { - Lack of confidence } & \text { - Falls/accidents } \\
\text { - Worry about disease } & \text { - Inability to play sports } \\
\begin{array}{l}\text { and future/fear of } \\
\text { disease progressing }\end{array} & \begin{array}{c}\text { or engage in physical } \\
\text { activities }\end{array} \\
\text { - Poor spatial } & \text { - Difficulty recognizing } \\
\text { perception and } & \text { faces } \\
\text { mobility } & \text { - Embarrassment } \\
\text { - Dependency on other } & \text { - Disorientation } \\
\text { people } & \text { - Vision slow to adjust in } \\
\text { - Difficulty completing } & \text { morning } \\
\begin{array}{l}\text { activities of daily } \\
\text { living }\end{array} & \end{array}$ \\
\hline
\end{tabular}

Fig. 4 Final dry AMD conceptual model. Bold text: Salient symptoms/impacts were defined as those which were mentioned by $\geq 50 \%$ of patients and received a disturbance rating of $\geq 5$ on average. $A M D$ age-related macular degeneration. ${ }^{a}$ Concepts outside the saliency

relevant or important effects that changes in visual acuity have on aspects of life they consider important [9]. Evidence obtained from patients with wet AMD suggest that general QoL instruments are inferior to vision-specific instruments when measuring health-related QoL in patients with visual disorders [9].

The NEI-VFQ-25, often considered a goldstandard patient-reported outcome instrument for ophthalmology conditions, is frequently incorporated into ophthalmology studies definition, but included due to proximity of mentions and disturbance to the salient concepts

submitted to regulatory and health technology assessment authorities for review. However, the NEI-VFQ-25 focuses predominantly on measuring the impacts, not the symptoms, of ophthalmic disease. Although the salient impacts in our conceptual model are similar to impacts measured by the NEI-VFQ-25, many of the concepts in our final conceptual model are not measured by either generic or available visionspecific tools. Specifically, most vision-specific instruments, such as the NEI-VFQ-25, that are 
used to assess QoL in patients with AMD cover few, if any, of the symptoms identified in our final conceptual model.

A strength of this study is the use of a threestep, systematic approach that follows FDA recommendations to obtain information directly from patients. However, all physicians and patients recruited for interviews were from the United States and therefore may not reflect the patient dry AMD experience globally. Although patients participating in the study had a physician-confirmed diagnosis of dry AMD and provided information on visual acuity, information was not collected on other disease features such as drusen or geographic atrophy. While this could be viewed as a limitation of this research, it is an inherent limitation of conceptual models that aim to balance model generalizability with granularity. Additionally, the purpose of this research was not to develop a conceptual model for a particular dry AMD phenotype, but rather a general model of the patient experience in order to inform future development and selection of PROMs. The interviews performed in this study were reliant on patient recall to identify signs, symptoms, and impacts, and therefore could be affected by recall and recency bias. Lastly, the conceptual model was developed using results from a moderate number of patients. However, concept saturation was achieved, and no new concepts were elicited during later interview waves.

\section{CONCLUSIONS}

To better understand the dry AMD patient experience, we captured, defined, and organized information on the distinct signs, symptoms, and impacts important to patients with dry AMD using a methodology that rigorously adhered to guidance developed by the FDA. The dry AMD conceptual model presented herein includes results obtained from a targeted literature review that were refined and validated through clinician and patient interviews.

Thirty-five signs, symptoms, and impacts of dry AMD were identified, 19 of which were mentioned by at least $50 \%$ of patients. As the first known conceptual model for dry AMD, the inclusion of signs, symptoms, and immediate and general impacts will likely aid in the development of dry AMD-specific patient-reported outcomes instruments reflecting a unique dry AMD patient experience. Furthermore, it offers an opportunity to better understand how a treatment for dry AMD may benefit patient QoL.

\section{ACKNOWLEDGEMENTS}

The authors wish to acknowledge and thank the study participants for their contributions to this study.

Funding. This study and the journal's publication fees were funded by Astellas Pharma, Inc.

Medical Writing and Editorial Assistance. Medical writing (Beth Lesher, PharmD, BCPS, of Pharmerit International, Bethesda, MD) and editorial support (Regina Switzer, PhD, and Elizabeth Hermans, PhD, of OPEN Health Medical Communications, Chicago, IL) were funded by the study sponsor.

Authorship. All named authors meet the International Committee of Medical Journal Editors (ICMJE) criteria for authorship for this article, take responsibility for the integrity of the work as a whole, and have given their approval for this version to be published.

Authorship Contributions. NMS directed the study and is responsible for study design decisions. All authors (NMS, LB-M, JS, and LG) made substantive contributions to the study design, analysis, and interpretation. All authors (NMS, LB-M, JS, and LG) critically reviewed the manuscript and provided final approval.

Disclosures. NMS and JS are employees of Astellas Pharma, Inc. LG is an employee of IQVIA. LB-M is an independent researcher but was an employee of IQVIA at the time of this research. 
Compliance with Ethics Guidelines. Ethics approval was obtained from the New England Institutional Review Board (NEIRB), 197 First Avenue, Suite 250, Needham, MA 02494. The study was conducted in accordance with ethical principles that have their origin in the Declaration of Helsinki and are consistent with Good Clinical Practice and applicable regulatory requirements. The study was conducted in accordance with the regulations of the United States FDA as described in 21 CFR 50 and 56, applicable laws, and institutional review board requirements.

Data Availability. The datasets generated during and/or analyzed during the current study are available from the corresponding author on reasonable request.

Open Access. This article is licensed under a Creative Commons Attribution-NonCommercial 4.0 International License, which permits any non-commercial use, sharing, adaptation, distribution and reproduction in any medium or format, as long as you give appropriate credit to the original author(s) and the source, provide a link to the Creative Commons licence, and indicate if changes were made. The images or other third party material in this article are included in the article's Creative Commons licence, unless indicated otherwise in a credit line to the material. If material is not included in the article's Creative Commons licence and your intended use is not permitted by statutory regulation or exceeds the permitted use, you will need to obtain permission directly from the copyright holder. To view a copy of this licence, visit http://creativecommons.org/licenses/by$\mathrm{nc} / 4.0 /$.

\section{REFERENCES}

1. Bourne RRA, Stevens GA, White RA, Smith JL, Flaxman SR, Price $\mathrm{H}$, et al. Causes of vision loss worldwide, 1990-2010: a systematic analysis. Lancet Glob Health. 2013;1(6):e339-49.
2. Pascolini D, Mariotti SPM. Global data on visual impairment. 2010. https://www.who.int/blindness/ publications/globaldata/en/. Accessed 11 Feb 2020.

3. Wong WL, Su X, Li X, Cheung CMG, Klein R, Cheng C-Y, et al. Global prevalence of age-related macular degeneration and disease burden projection for 2020 and 2040: a systematic review and meta-analysis. Lancet Glob Health. 2014;2(2): e106-16.

4. Cook HL, Patel PJ, Tufail A. Age-related macular degeneration: diagnosis and management. Br Med Bull. 2008;85:127-49.

5. Flaxel CJ, Adelman RA, Bailey ST, Fawzi A, Lim JI, Vemulakonda GA, et al. Age-related macular degeneration preferred practice pattern. Ophthalmology. 2020;127(1):P1-65.

6. Taylor DJ, Hobby AE, Binns AM, Crabb DP. How does age-related macular degeneration affect realworld visual ability and quality of life? A systematic review. BMJ Open. 2016;6(12):e011504.

7. Food and drug administration. Patient-focused drug development: collecting comprehensive and representative input. Guidance for industry. Food and drug administration staff, and other stakeholders. 2020. https://www.fda.gov/media/113653/down load. Accessed 23 July 2020.

8. Mitchell J, Bradley C. Quality of life in age-related macular degeneration: a review of the literature. Health Qual Life Outcomes. 2006;21(4):97.

9. Yuzawa M, Fujita K, Tanaka E, Wang EC. Assessing quality of life in the treatment of patients with agerelated macular degeneration: clinical research findings and recommendations for clinical practice. Clin Ophthalmol. 2013;7:1325-32.

10. Food and Drug Administration. Guidance for industry: patient-reported outcome measures: use in medical product development to support labeling claims. 2009. https://www.fda.gov/media/ 77832/download. Accessed 13 Feb 2020.

11. Patrick DL, Burke LB, Gwaltney CJ, Leidy NK, Martin ML, Molsen E, et al. Content validityestablishing and reporting the evidence in newly developed patient-reported outcomes (PRO) instruments for medical product evaluation: ISPOR PRO Good Research Practices Task Force report: part 2-assessing respondent understanding. Value Health. 2011;14(8):978-88.

12. Patrick DL, Burke LB, Gwaltney CJ, Leidy NK, Martin ML, Molsen E, et al. Content validityestablishing and reporting the evidence in newly developed patient-reported outcomes (PRO) instruments for medical product evaluation: ISPOR 
PRO good research practices task force report: part 1-eliciting concepts for a new PRO instrument. Value Health. 2011;14(8):967-77.

13. Lastacaft [package insert]. Madison, NJ, USA: Allergan. 2020.

14. Ikervis [Summary of Product Characteristics]. Tampere, Finland: Santen Oy. 2020.
15. Lucentis [Summary of Product Characteristics]. Dublin 4, Ireland: Novartis Europharm Limited. 2016.

16. Braithwaite T, Calvert M, Gray A, Pesudovs K, Denniston AK. The use of patient-reported outcome research in modern ophthalmology: impact on clinical trials and routine clinical practice. Patient Relat Outcome Meas. 2019;10:9-24. 